\title{
Introduction to the GaLA Conf 2019 Special Issue
}

\author{
Antonios Liapis ${ }^{1}$, Georgios N. Yannakakis ${ }^{1}$, Manuel Gentile ${ }^{2}$, Manuel Ninaus ${ }^{3}$ \\ ${ }^{I}$ Institute of Digital Games, University of Malta, Malta, \\ \{antonios.liapis;georgios.yannakakis\}@um.edu.mt \\ ${ }^{2}$ Institute for Educational Technology, National Research Council of Italy, Palermo, \\ Italy,manuel.gentile@itd.cnr.it \\ ${ }^{3}$ Department of Psychology, University of Innsbruck, Austria
}

It is a great pleasure for us to introduce this issue of the IJSG, which is dedicated to the Games and Learning Alliance Conference (GaLA Conf) that was held in Athens, November 27-29, 2019. Almost 70 participants converged in this beautiful, historic city in order to share knowledge and experiences related to serious games and gamification, their techniques and their application.

A number of the best conference papers were selected, and the authors were invited to extend their paper with at least one-third new content, and to submit their paper to IJSG for a regular peer-review process. This issue contains three of the resulting articles, which gives an overview of the rich field of serious games.

The first article, by Szabina Fodor and Balázs Barna, is titled "An empirical study on factors affecting user engagement in a gamified team building environment" [1]. The authors presented a study on data retrieved from a gamified team building application, which promotes employees' joint sport activities. The aim was to identify and evaluate behavior usage patterns, and user engagement indicators based on interaction data gained from the application and to determine critical factors affecting engagement levels. The study revealed that behavior patterns among users of a gamified service can be distinguished first by their usage time and then by additional influencing factors beyond personal behavioral patterns such as 'corporate culture' and the importance of the 'organizers'.

The second article by Roland Klemke, Alessandra Antonaci, and Bibeg Limbu, titled "Designing and Implementing Gamification: GaDeP, Gamifire, and applied Case Studies" [2], introduces the Gamification Design Process (GaDeP) as a design methodology to apply the concept of meaningful gamification. To validate $\mathrm{GaDeP}$ as a design framework for the creation of gamified applications independent of specific applications, the authors followed a two-fold approach. Using GaDeP, the Gamefire platform was designed to be a platformindependent, cloud-based infrastructure for meaningful gamification of massive open online courses (MOOCs). Second, the authors proved the efficacy of GaDeP beyond the use of a MOOC and reported a set of interesting case studies.

The last paper by Simon Greipl, Korbinian Moeller, Kristian Kiili and Manuel Ninaus, titled "Different performance, full experience: a learning game applied throughout adulthood" [3], evaluated the applicability of educational games across the lifespan. The authors examined age-related differences along three domains: cognition (as measured by in-game performance), player experience with the game, and general player attitudes towards technology. The results of this study revealed that players' experience with the game and overall attitudes towards technology appeared comparable across the lifespan. With regards to cognition, the performance metrics related to speed reflected an age-related decline, while error-metrics were associated with educational background. Based on the results, the authors provided design recommendations for educational games when used across the lifespan. 
pag. 60

After the presentation of the interesting and relevant articles, we would now like to draw your attention to the next edition of the GaLA Conference, the first virtual edition of GaLA conferences, which will take place in the Laval Virtual World during 9-10 December 2020 (https://conf.seriousgamessociety.org/). The conference will be another great opportunity to share knowledge, opinions and ideas and advance the field of serious games. The conference will also include an exhibition where developers can showcase their latest products. We hope to meet you all, even if virtually, at the GaLA Conference in Laval.

Antonios Liapis

Georgios N. Yannakakis

Manuel Gentile

Manuel Ninaus

\section{References}

[1] S. Fodor and B. Barna, An empirical study on factors affecting user engagement in a gamified team building environment, The International Journal of Serious Games, Vol. 8, No. 3, September 2020.

[2] R. Klemke, A. Antonaci, and B. Limbu, Designing and Implementing Gamification: GaDeP, Gamifire, and applied Case Studies, The International Journal of Serious Games, Vol. 8, No. 3, September 2020.

[3] S. Greipl, K. Moeller, K. Kiili and M. Ninaus, Different performance, full experience: a learning game applied throughout adulthood, The International Journal of Serious Games, Vol. 8, No. 3, September 2020. 